\title{
Relevância da pré-hipertensão como categoria diagnóstica em adultos assintomáticos
}

\author{
Relevance of prehypertension as a diagnostic category in asymptomatic adults
}

\author{
Fernando Costa Nary ${ }^{1}$, Raul D. Santos ${ }^{1}$, Antonio Gabriele Laurinavicius ${ }^{1}$, Raquel Dilguerian de Oliveira Conceição, \\ José Antonio Maluf de Carvalho ${ }^{1}$
}

\begin{abstract}
RESUMO
Objetivo: Avaliar a associação da pré-hipertensão com perfis metabólico, inflamatório e de risco cardiovascular em indivíduos assintomáticos. Métodos: Entre 2006 a 2009, 11.011 adultos assintomáticos (média de idade de 43 anos; $22 \%$ mulheres) foram submetidos a protocolo de check-up, sendo classificados em 3 grupos: normotensos (pressão arterial $<120 \times 80 \mathrm{mmHg}$ ), pré-hipertensos (pressão arterial= $120 / 80 \mathrm{mmHg}$ e $<140 / 90 \mathrm{mmHg}$ ) e hipertensos (pressão arterial $\geq 140 / 90 \mathrm{mmHg}$ ou diagnóstico prévio de hipertensão arterial). Foram avaliados os perfis metabólico e de risco cardiovascular de cada um dos três grupos. Resultados: A prevalência de normotensão, préhipertensão e hipertensão foi, respectivamente, de 27,9\%, 53,9\% e 18,2\%. Quando comparados com os indivíduos normotensos, os pré-hipertensos apresentaram média de idade maior $(42,7$ versus 40 anos; $\mathrm{p}<0,001$ ), maior índice de massa corporal (média: $26,7 \mathrm{~kg} / \mathrm{m}^{2}$ versus $\left.24 \mathrm{~kg} / \mathrm{m}^{2} ; \mathrm{p}<0,001\right)$, níveis de triglicérides mais elevados (média: 139mg/dL versus 108mg/dL; $\mathrm{p}<0,001$ ), níveis de LDL-colesterol mais elevados (média: $128 \mathrm{mg} / \mathrm{dL}$ versus $117 \mathrm{mg} / \mathrm{dL}$; $\mathrm{p}<0,001$ ) e níveis séricos menores de HDL-colesterol (média: $46,7 \mathrm{mg} / \mathrm{dL}$ versus $52,7 \mathrm{mg} / \mathrm{dL} ; \mathrm{p}<0,001)$. Os pré-hipertensos apresentaram maior prevalência de alterações na glicemia de jejum (OR: 1,69; IC95\%: $1,39-2,04)$, sobrepeso e obesidade - ou seja, índice de massa corporal $>25 \mathrm{~kg} / \mathrm{m}^{2}$ (OR: 2,48; IC95\%: 2,24-2,74), esteatose hepática (OR: 2,23; IC95\%: 1,97-2,53), síndrome metabólica (OR: 3,05; IC95\%: 2,67-3,49) e níveis $>2 \mathrm{mg} / \mathrm{L}$ de proteína C-reativa de alta sensibilidade (OR: 1,52; IC95\%: 1,35-1,71). Conclusão: A pré-hipertensão está associada a aumento da prevalência de síndrome metabólica, esteatose hepática e inflamação subclínica.
\end{abstract}

Descritores: Pré-hipertensão; Fatores de risco; Doenças cardiovasculares; Inflamação; Proteína C-reativa; Síndrome x metabólica

\section{ABSTRACT}

Objective: To assess the association of prehypertension with metabolic, inflammatory and cardiovascular risk profile in asymptomatic individuals.
Methods: Between 2006 and 2009, 11,011 asymptomatic adults (mean age: 43 years; 22\% females), underwent a check-up protocol. They were divided into 3 groups: normotensive group (arterial pressure $=120 / 80 \mathrm{mmHg}$ ), prehypertensive group (arterial pressure $>120 / 80 \mathrm{mmHg}$ and $<140 / 90 \mathrm{mmHg}$ ) and hypertensive group (arterial pressure $\geq 140 / 90 \mathrm{mmHg}$ or prior diagnosis of hypertension). Each group metabolic and cardiovascular group profile was assessed. Results: The prevalence of normotension, prehypertension and hypertension was $27.9 \%, 53.9 \%$ and $18.2 \%$, respectively. Prehypertensive individuals were older (mean age: 42.7 versus 40 years; $p<0.001$ ) than normotensive patients, and had higher body mass index (mean: $26.7 \mathrm{~kg} / \mathrm{m}^{2}$ versus $24 \mathrm{~kg} / \mathrm{m}^{2} ; \mathrm{p}<0.001$ ), higher plasma triglycerides levels (mean: $139 \mathrm{mg} / \mathrm{dL}$ versus 108mg/dL; $\mathrm{p}<0.001$ ), higher LDL-choleterol levels (mean: $128 \mathrm{mg} / \mathrm{dL}$ versus $117 \mathrm{mg} / \mathrm{dL} ; \mathrm{p}<0.001$ ), and lower HDL-cholesterol (mean: $46.7 \mathrm{mg} / \mathrm{dL}$ versus $52.7 \mathrm{mg} / \mathrm{dL} ; \mathrm{p}<0.001$ ). Prehypertensive individuals were more likely to have impaired fasting glucose (OR: 1.69; 95\% Cl: 1.39-2.04), overweight and obesity - body mass index $>25 \mathrm{~kg} / \mathrm{m}^{2}$ (OR: $2.48 ; 95 \% \mathrm{Cl}: 2.24-2.74$ ), hepatic steatosis: (OR: 2.23; 95\% Cl: 1.97-2.53), metabolic syndrome (OR: 3.05; 95\%Cl: 2.67-3.49), and high-sensitivity C-reactive protein levels $>2 \mathrm{mg} / \mathrm{L}$ (OR: 1.52; 95\% Cl: 1.35-1.71). Conclusion: Prehypertension is associated with an increased prevalence of metabolic syndrome, hepatic steatosis and subclinical inflammation.

Keywords: Prehypertension; Risk factors; Cardiovascular diseases; Inflammation; C-reactive protein; Metabolic syndrome x

\section{INTRODUÇÃo}

A hipertensão arterial sistêmica (HAS) constitui um dos principais fatores de risco cardiovascular modificáveis, além de importante problema de saúde pública. A mortalidade por doença cardiovascular aumenta progressivamente com a elevação da pressão arterial (PA) a partir de $115 / 75 \mathrm{mmHg}$ de forma linear, contínua e inde-

Trabalho realizado no Hospital Israelita Albert Einstein, São Paulo, SP, Brasil; Instituto do Coração, Hospital das Clínicas, Faculdade de Medicina, Universidade de São Paulo, São Paulo, SP, Brasil.

1 Hospital Israelita Albert Einstein, São Paulo, SP, Brasil.

Autor correspondente: Raul D. Santos - Avenida Albert Einstein, 627/701 - Morumbi - CEP: 05652-900 - São Paulo, SP, Brasil - Tel.: (11) 2151-1000 - E-mail: raul.filho@einstein.br

Data de submissão: 17/7/2013 - Data de aceite: 3/9/2013

Conflito de interesse: não há. 
pendente $^{(1,2)}$. Em 2001, cerca de 7,6 milhões de mortes no mundo foram atribuídas à elevação da PA, sendo a maioria em países de baixo e médio desenvolvimento econômico e mais da metade em indivíduos entre 45 e $69 \operatorname{anos}^{(3,4)}$.

O termo "pré-hipertensão" corresponde a valores de $\mathrm{PA} \geq 120 / 80 \mathrm{mmHg}$ e $<140 / 90 \mathrm{mmHg}$ e foi originalmente introduzido em 2003, em ocasião da publicação do VII Joint National Committee on Prevention, Detection, Evaluation and Treatment of High Blood Pressure (JNC) com o objetivo de identificar uma categoria de indivíduos com valores limítrofes de PA, e, desse modo, sob maior risco de apresentar HAS no futuro ${ }^{(4)}$. Demonstrou-se que, em relação aos normotensos, indivíduos pré-hipertensos apresentam um risco duas vezes maior de evoluir para $\mathrm{HAS}^{(5)}$ e, ao mesmo tempo, um risco cardiovascular significativamente mais elevado ${ }^{(6)}$.

Apesar disso, o rótulo de pré-hipertensão não foi universalmente aceito como categoria diagnóstica, não sendo adotado nas VI Diretrizes Brasileiras de $\mathrm{HAS}^{(2)}$. Críticos da nomenclatura apontam que o diagnóstico de pré-hipertensão identifica um grupo heterogêneo de indivíduos predominantemente saudáveis, com diferentes graus de risco de eventos cardiovasculares e de evolução para $\operatorname{HAS}^{(7)}$. Muitos desses indivíduos saudáveis poderiam ser estigmatizados como doentes e tratados com medidas farmacológicas desnecessárias $^{(8)}$. Por outro lado, o diagnóstico de pré-hipertensão pode constituir ferramenta valiosa de rastreamento e de sensibilização individual para a adesão a um estilo de vida saudável. A pré-hipertensão é o ponto de convergência de vários fatores de risco cardiovascular, fato que reforça a importância de se adotar a nomenclatura na prática clínica diária, com o intuito de facilitar a identificação e a sensibilização de indivíduos candidatos a ações de modificação do estilo de vida.

\section{OBJETIVO}

Caracterizar o perfil metabólico e de risco cardiovascular da pré-hipertensão em uma população de adultos brasileiros assintomáticos, avaliando sua associação com fatores de risco tradicionais, síndrome metabólica, esteatose hepática, resposta hiper-reativa da pressão arterial ao exercício e inflamação subclínica.

\section{MÉTODOS}

Foram avaliados retrospectivamente 11.011 adultos brasileiros (média de idade de 43 anos, sendo $22 \%$ do gênero feminino) submetidos ao protocolo de revisão periódica de saúde do Hospital Israelita Albert Einstein durante o período de 2006 a 2009. O protocolo fazia parte de programas de saúde de empresas privadas da cidade de São Paulo, era obrigatório e pago pelos empregadores. Como parte do protocolo, realizaram-se extensas avaliações clínica e laboratorial, ultrassonografia abdominal e teste ergométrico de rotina. A aferição da PA sistólica (PAS) e diastólica (PAD) foi realizada em cada indivíduo, segundo as diretrizes da American Heart Association $^{(9)}$, usando-se um esfigmomanômetro aneroide, devidamente aferido e calibrado, e com manguito adequado a circunferência do braço. A PA foi medida três vezes durante a avaliação, sendo utilizado um valor médio. Os participantes do estudo foram classificados em três grupos, de acordo com a medida da PA: normoten$\operatorname{sos}(<120 / 80 \mathrm{mmHg})$, pré-hipertensos $(\geq 120 / 80 \mathrm{mmHg}$ e $<140 / 90 \mathrm{mmHg}$ ) e hipertensos $(\geq 140 / 90 \mathrm{mmHg}$ ou diagnóstico prévio de hipertensão arterial).

Este estudo foi aprovado pelo comitê de ética do Hospital Israelita Albert Einstein (CAAE n. o 0094.0.028.000-09), tendo sido dispensado do termo de consentimento.

\section{Variáveis clínicas}

Foram excluídos do estudo indivíduos diabéticos e com diagnóstico de doença cardiovascular prévio. Os participantes foram interrogados em relação à presença prévia de dislipidemias, HAS (diagnóstico prévio HAS, uso de medicamentos anti-hipertensivos ou PA medida $\geq 140 / 90 \mathrm{mmHg}$ ), diabetes (uso prévio de medicação para diabetes ou glicemia de jejum $>126 \mathrm{mg} / \mathrm{dL}$ ) e tabagismo (consumo de pelo menos um cigarro nos últimos 30 dias). Foi calculado o índice de massa corporal (IMC) pela fórmula peso/altura ${ }^{2}\left(\mathrm{~kg} / \mathrm{m}^{2}\right)$ para classificação dos indivíduos em eutróficos (IMC <25), com sobrepeso $(25-29,9)$ e obesos $(\geq 30)$. Foi medida a circunferência abdominal, para avaliação da adiposidade visceral. Calculou-se o nível de atividade física (sedentarismo, pouco ativo, ativo e muito ativo) de acordo com os critérios do Questionário Internacional de Atividade Física (IPAQ, sigla do inglês International Physical Activity Questionnaire $)^{(10)}$. Para uma melhor avaliação, os sedentários e pouco ativos foram agrupados num grupo de baixa atividade física e os ativos em muito ativos foram agrupados num grupo ativo. $\mathrm{O}$ consumo de álcool foi quantificado pelo questionário AUDIT (sigla do inglês Alcohol Use Disorders Identification Test) ${ }^{(11)}$, sendo considerado consumo moderado/elevado quando escore $\geq 8$.

\section{Variáveis laboratoriais}

Exames de bioquímica clínica foram realizados após jejum de pelo menos 12 horas. Colesterol total, HDL-colesterol (HDL-c), triglicérides (TG) e glice- 
mia foram determinados por métodos enzimáticos em plataforma Vitros ${ }^{\circledR}$ (Ortho-Clinical Diagnostics, Inc., Johnson \& Johnson, Nova York, EUA). O LDL-colesterol (LDL-c) foi calculado pela fórmula de Friedwald para $\mathrm{TG}<400 \mathrm{mg} / \mathrm{dL}$. Foram considerados glicemia de jejum alterada valores entre $100 \mathrm{mg} / \mathrm{dL}$ e $126 \mathrm{mg} / \mathrm{dL}$. Os valores de proteína C-reativa de alta sensibilidade (PCR-as) foram determinados por imunoturbidimetria (Dade-Boehring, EUA). A presença de inflamação subclínica foi considerada para valores de PCR-as $>2,0 \mathrm{mg} / \mathrm{L}$.

\section{Teste ergométrico para pesquisa de resposta hiper- reativa da pressão arterial ao exercício}

Foi realizado teste ergométrico em esteira rolante pelo protocolo de Ellestad, sendo o mesmo interrompido por cansaço físico. Considerou-se resposta hiper-reativa da PA o registro de valores de PAS $>220 \mathrm{mmHg}$ e/ou a elevação de $15 \mathrm{mmHg}$ ou mais na $\mathrm{PAD}$, partindo-se de valores normais de PA em repouso.

\section{Ultrassografia abdominal para pesquisa de esteatose hepática}

A ultrassonografia abdominal foi realizada em todos os pacientes após, no mínimo, 6 horas em jejum. A esteatose hepática foi definida por um padrão ultrassonográfico de fígado brilhante com evidência de contraste entre os parênquimas hepático e renal, utilizando método descrito previamente ${ }^{(12)}$. Todos os exames foram realizados num equipamento ACUSON XP-10 (Siemens Company, Mountain View, EUA).

\section{Avaliação da presença da síndrome metabólica e do risco cardiovascular estimado}

A síndrome metabólica foi diagnosticada de acordo com os critérios da International Diabetes Federation (IDF) ${ }^{(13)}$. O risco de morte cardíaca e infarto do miocárdio em 10 anos foi calculado pelo escore de Framingham de acordo com o gênero ${ }^{(14)}$. Os participantes foram agrupados como sendo de baixo risco (risco $<10 \%$ ) ou de risco médio/alto ( $\geq 10 \%)$.

\section{Análise estatística}

Todas as variáveis foram analisadas e comparadas estatisticamente entre os grupos de pré-hipertensos e normotensos. Foram descritas as medidas qualitativas com uso de frequências relativas e absolutas segundo grupos (normotenso e pré-hipertensos) e verificou-se a existência de associação dessas medidas entre os grupos por meio do teste do $\chi^{2}$. As medidas quantitativas foram descritas segundo classificação com uso de medidas resu- mo (média \pm desvio padrão), exceto para variáveis com distribuição anormal descritas como medianas (mínimo; máximo). As comparações foram realizadas pelo teste $t$ para duas amostras ou teste de Mann-Whitney para variáveis não paramétricas. A associação da pré-hipertensão a fatores de risco cardiovascular e parâmetros metabólicos foi realizada por análise multivariada, ajustando-se inicialmente para gênero e idade por modelos lineares gerais. Posteriormente, todas as variáveis avaliadas foram testadas conjuntamente em um modelo de regressão multinomial múltipla. Foram calculados os odds ratios (OR) com os respectivos intervalos de confiança 95\% (IC95\%) para cada variável do modelo, tendo o grupo de pré-hipertensos como referência. Para verificar a relação contínua entre valores da PA e a PCR-as, foram criados diagramas de dispersão e ajustados modelos lineares generalizados, com distribuição gama e função de ligação identidade. Os testes foram realizados com nível de significância de 5\%.

\section{RESULTADOS}

A figura 1 mostra a prevalência de normotensão, préhipertensão e hipertensão arterial no grupo estudado. A maioria da população $(54 \%$; $n=5.946)$ apresentava pré-hipertensão. As figuras 2 e 3 mostram a prevalência de acordo com o gênero, sendo a pré-hipertensão mais frequente nos homens.

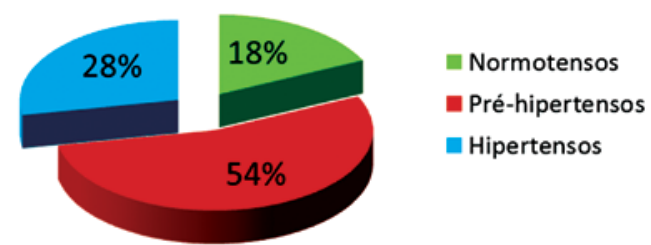

Figura 1. Prevalência de pré-hipertensão

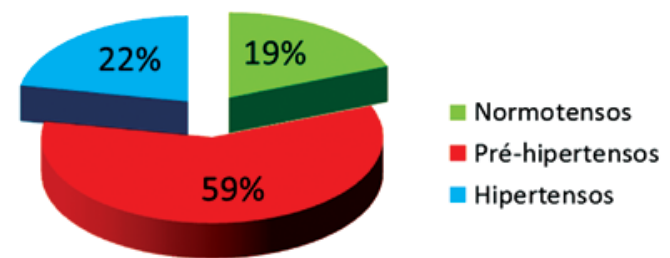

Figura 2. Prevalência de pré-hipertensão entre os homens

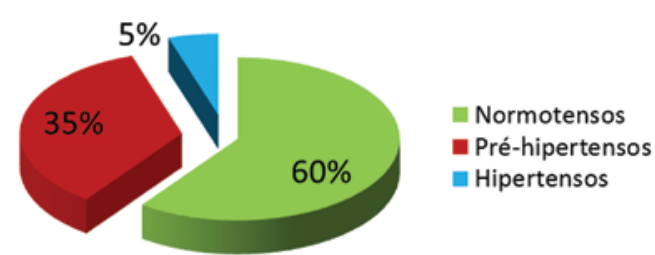

Figura 3. Prevalência de pré-hipertensão entre as mulheres 
A tabela 1 mostra a comparação das diversas variáveis clínicas e laboratoriais entre os portadores de normotensão e pré-hipertensão. Quando comparados com os indivíduos normotensos, os pré-hipertensos apresentaram maior IMC, maiores valores de circunferência abdominal e, consequentemente, maior prevalência de sobrepeso e obesidade.

Não houve diferença em relação à prevalência de tabagismo entre os grupos $(10,3 \%$ versus $11,6 \%$, respectivamente, para normotensos e pré-hipertensos; $p=0,122$ ).

Em relação ao grau de atividade física, houve um maior número de indivíduos com baixa atividade física no grupo de pré-hipertensos em relação aos normotensos (respectivamente 60,6\% e 59,4\%; p<0,001). Da mesma forma, houve maior prevalência de indivíduos com consumo moderado/elevado de álcool no grupo de pré-hipertensos $(14,2 \%$ versus $9,1 \%$; $\mathrm{p}<0,001)$.

Tabela 1. Variáveis demográficas, clínicas, hemodinâmicas e laboratoriais entre os grupos de normotensos e pré-hipertensos

\begin{tabular}{lccc}
\hline Variáveis & $\begin{array}{c}\text { Pré-hipertensos } \\
\text { (n=5.932) } \\
\text { Valores médios } \\
\text { (DP) }\end{array}$ & $\begin{array}{c}\text { Normotensos } \\
\text { (n=3.076) } \\
\text { Valores médios } \\
\text { (DP) }\end{array}$ & $\begin{array}{c}\text { Valor } \\
\text { de p }\end{array}$ \\
\hline Idade (anos) & $42,7(7,3)$ & $40(6,54)$ & $<0,001$ \\
Gênero (\% mulheres) & 14 & 47 & $<0,001$ \\
IMC (kg/m²) & $27(3,7)$ & $24(3,2)$ & $<0,001$ \\
Colesterol total (mg/dL) & $201(37,8)$ & $191(35,6)$ & $<0,001$ \\
Triglicérides* (mg/dL) & $92(20-840)$ & $118(18-3.133)$ & $<0,001$ \\
LDL-colesterol (mg/dL) & $128(33,9)$ & $117(32,8)$ & $<0,001$ \\
HDL-colesterol (mg/dL) & $47(12)$ & $53(14)$ & $<0,001$ \\
Gama-GT* (U/L) & $30(7-379)$ & $22(5-375)$ & $<0,001$ \\
Glicemia de jejum (mg/dL) & $91(15,3)$ & $86(1,9)$ & $<0,001$ \\
PCR-as* (mg/L) & $1(0,1-10)$ & $1,2(0,1-10)$ & $<0,001$ \\
Tabagismo (\%) & 11,6 & 10,3 & 0,122 \\
Sedentarismo (IPAQ) (\%) & 25,5 & 23,8 & $<0,001$ \\
Consumo de álcool AUDIT>8 (\%) & 14,2 & 9,1 & $<0,001$ \\
Escore de Framingham > 10\% & 8 & 3 & $<0,001$ \\
\hline * Medianas. & & & \\
DP: desvio padrāo; IMC: índice de massa corporal; PCR-as: proteína C-reativa de alta sensibilidade; IPAQ: International \\
Physical Activity Questionnaire; AUDIT: Alcohol Use Disorders /dentification Test.
\end{tabular}

A tabela 1 mostra também que os pré-hipertensos apresentaram valores mais elevados de LDL-c, TG e valores mais baixos de HDL-c $(p<0,001)$. O mesmo ocorreu com a glicemia de jejum e a prevalência de glicemia de jejum alterada $12 \%$ versus $5 \%(\mathrm{p}<0,001)$. Consequentemente aos achados clínicos e laboratoriais, tanto a prevalência da síndrome metabólica (32\% versus $11 \%$; $\mathrm{p}<0,001)$ como o número de indivíduos com risco médio/alto para doença coronária (8\% versus $3 \%$; $\mathrm{p}<0,001)$ foram maiores entre os pré-hipertensos. Os portadores de pré-hipertensão apresentaram maior prevalência de esteatose hepática e resposta hiper-reativa da PA ao teste ergométrico $(p<0,001$ para ambas as variáveis). As medianas da PCR-as foram mais elevadas nos pré-hipertensos $(p<0,001)$ e, consequentemente, houve maior prevalência de indivíduos com inflamação subclínica ( $29 \%$ versus $23 \%$; $\mathrm{p}<0,001)$ nesse grupo.

A tabela 2 mostra os odds ratio para alterações metabólicas, hemodinâmicas e inflamatórias entre os pré-hipertensos e normotensos após ajuste para idade e gênero.

A tabela 3 mostra esses parâmetros no modelo ajustado para todos os fatores de confusão. Após ajustes, os pré-hipertensos permaneceram com maiores valores de LDL-c, maior prevalência de HDL-c baixo, alterações na glicemia de jejum, síndrome metabólica, PA alterada no teste ergométrico, esteatose hepática e inflamação subclínica $(\mathrm{p}<0,05)$. Contudo, apesar dos achados, após ajuste para a idade, não houve diferença entre os grupos na prevalência de indivíduos com risco médio/alto

Tabela 3. Odds ratio para alterações metabólicas, inflamatórias e hemodinâmicas entre normotensos e pré-hipertensos em modelo multivariado

\begin{tabular}{lccc}
\hline Variáveis & OR & IC95\% & Valor de p \\
\hline IMC $>25$ & 1,66 & $1,46-1,89$ & $<0,001$ \\
PCR-as $>2 \mathrm{mg} / \mathrm{L}$ & 1,21 & $1,06-1,38$ & 0,006 \\
ERF médio/alto $(>10 \%)$ & 0,94 & $0,7-1,26$ & 0,662 \\
Síndrome metabólica & 2,14 & & $<0,001$ \\
Esteatose hepática & 1,35 & $1,16-1,58$ & $<0,001$ \\
RHR da PA & 4,05 & $2,87-5,71$ & $<0,001$ \\
\hline
\end{tabular}

IC95\%: intervalo de confiança de 95\%; IMC: índice de massa corporal; PCR-as: proteína C-reativa de alta sensibilidade; ERF: Escore de Risco de Framingham; RHR da PA: resposta hiper-reativa da pressão arterial ao teste ergométrico; OR: odds ratio.

Tabela 2. Prevalência de síndrome metabólica, glicemia de jejum alterada, esteatose hepática, inflamação subclínica e resposta hiper-reativa da pressão arterial no teste ergométrico, com odds ratio ajustados para gênero e idade

\begin{tabular}{lcccc}
\hline Variáveis & Pré-hipertensos (\%) & Normotensos (\%) & Valor de p & OR (IC95\%) \\
\hline Síndrome metabólica & 31,6 & 11,1 & $<0,001$ & $3,05(2,67-3,49)$ \\
Glicemia de jejum alterada & 11,8 & 4,8 & $<0,001$ & $1,69(1,39-2,04)$ \\
Esteatose hepática & 35,9 & 13,8 & $<0,001$ & $2,23(1,97-2,53)$ \\
Inflamação subclínica (PCR-as>2mg/L) & 29,2 & 23,1 & $<0,001$ & $1,52(1,35-1,71)$ \\
Hiper-reatividade da PA ao exercício & 9,8 & 1,6 & $<0,001$ & $5,06(3,68-6,95)$ \\
\hline
\end{tabular}

IC95\%: intervalo de confiança de 95\%; PCR-as: proténa C-reativa de alta sensibilidade; PA: pressão arterial; OR: odds ratio. 
pelo escore de Framingham. Finalmente, foi observado aumento de $0,018 \mathrm{mg} / \mathrm{L}$ e $0,025 \mathrm{mg} / \mathrm{L}$ nos níveis de PCR-as para cada aumento de $1 \mathrm{mmHg}$, respectivamente, na PAS e PAD ( $p<0,001)$, após ajuste para fatores de confusão.

\section{DISCUSSÃO}

Neste estudo, realizado em população oportunista de indivíduos assintomáticos submetidos à avaliação periódica de saúde, a pré-hipertensão arterial associou-se de forma independente, após ajuste para fatores de confusão, com um perfil metabólico desfavorável, maior prevalência de resposta hiper-reativa da PA ao teste ergométrico, esteatose hepática e inflamação subclínica. Nesta casuística, a pré-hipertensão afetou mais da metade da população estudada, sendo significativamente mais frequente em homens e em indivíduos sedentários e com excesso de peso. Previamente, em nosso meio, Cassani et al. e Silva et al. encontraram, em populações cerca de dez vezes menores do que a avaliada neste estudo, prevalências de pré-hipertensão de $45 \%$ e $36 \%$, respectivamente ${ }^{(15,16)}$. Da mesma forma, nesses estudos a pré-hipertensão associou-se ao gênero masculino, ao excesso de peso e ao baixo grau de atividade física.

A pré-hipertensão é considerada precursora da $\mathrm{HAS}^{(5,8)}$. A observação do comportamento da PA durante 50 anos, em 5.181 participantes do estudo de Framingham, revelou que os indivíduos classificados como pré-hipertensos evoluíram para o estágio de hipertensão arterial com maior frequência que os indivíduos normotensos ${ }^{(5)}$. A observação de um estudo prospectivo em indivíduos portadores de pré-hipertensão, que incluiu, em seu desenho, o desenvolvimento de HAS como um dos desfechos maiores, mostrou que, após 4 anos de seguimento, aproximadamente dois terços dos pré-hipertensos $(63,0 \%)$ evoluíram para o status de hipertensos ${ }^{(8)}$. Os achados deste estudo apontam a préhipertensão como preditor independente de resposta hiper-reativa da PA ao exercício. Sabe-se que a resposta hiper-reativa da PA ao teste ergométrico associa-se com o aparecimento de HAS em estudos prospectivos ${ }^{(17,18)}$. Possíveis explicações para tais achados seriam a presença de alteração na função endotelial e a maior rigidez vascu$\operatorname{lar}^{(19)}$. A associação independente aqui observada entre pré-hipertensão e resposta hiper-reativa da PA ao exercício reforça, portanto, o papel da pré-hipertensão como fator de risco para o posterior desenvolvimento de HAS.

A análise de vários estudos epidemiológicos revela que a pré-hipertensão está associada ao aumento do risco de desenvolvimento de doenças cardiovasculares. A análise prospectiva de um estudo coorte com 8.960 adultos de média idade, participantes do estudo
The Atherosclerosis Risk in Communities (ARIC) Study, mostrou que o risco relativo de incidência de doenças cardiovasculares foi $133 \%$ maior para os indivíduos com PA normal-alta quando comparados aqueles com PA ótima (PAS $<120 \mathrm{mmHg}$ e $\mathrm{PAD}<80 \mathrm{mmHg})^{(20)}$. Neste estudo, a pré-hipertensão associou-se com dislipidemia, disglicemia e inflamação subclínicas independentemente de sedentarismo, gênero, idade e excesso de peso. Estes dados sugerem que a pré-hipertensão possa associar-se a risco cardiovascular adicional àquele causado diretamente pelo aumento isolado da PA.

Atualmente, aceita-se que a aterosclerose seja um estado pró-inflamatório, sendo que valores elevados de PCR-as são marcadores independentes do risco de eventos cardiovasculares ${ }^{(21)}$. Em nossa casuística os portadores de pré-hipertensão tinham $21 \%$ mais chance de apresentar inflamação subclínica do que os normotensos. Fato importante foi a associação independente e linear da PA aos valores de PCR-as, confirmando a associação previamente descrita entre PA e inflamação ${ }^{(22)}$. Contudo, é importante enfatizar que tanto a PA como a inflamação são preditores independentes do risco de eventos cardiovasculares. Além do fato de a elevação da PA ser um estado pró-inflamatório, uma possível explicação para a maior elevação da PCR-as nos préhipertensos foi a maior prevalência de esteatose hepática nesses indivíduos, mesmo após ajuste para o peso e o consumo de álcool. Previamente demonstrou-se que a doença hepática não alcoólica, que engloba a esteatose hepática, associa-se de forma independente com a elevação da PCR-as ${ }^{(23)}$. Outro mecanismo fisiopatológico que pode estar associado aos achados deste estudo é a maior prevalência de resistência insulínica na população de pré-hipertensos. Embora não tenhamos medidos os modelos de homeostase (HOMA) a maior prevalência de disglicêmicos entre portadores de préhipertensão está de acordo com essa hipótese. De fato, estudos prévios associaram a resistência insulínica à elevação da PA, inflamação e esteatose hepática ${ }^{(24)}$. Da mesma forma, Toprak et al. ${ }^{(25)}$ encontraram associação positiva entre a pré-hipertensão e circunferência da cintura, glicemia de jejum e valores de insulina plasmáticos.

Os achados deste estudo são preocupantes em razão da alta prevalência de pré-hipertensão e de outros fatores associados encontrada em indivíduos relativamente jovens - o que foi contatado também por outros estudos realizados no Brasil ${ }^{(15,16)}$. Dessa forma, impõe-se fortemente a necessidade de modificações importantes no estilo de vida e em alguns casos de tratamento farmacológico ${ }^{(26)} \mathrm{dos}$ indivíduos nessa categoria de classificação da PA.

Uma limitação deste estudo foi seu desenho transversal, que não permitiu demonstrar causalidade, mas 
apenas associações. Da mesma forma, não é possível excluir um viés de seleção relativo à faixa socioeconômica da população estudada que, tendo acesso a uma rotina sistematizada de revisão periódica de saúde, poderia não ser representativa da população geral do Brasil. Contudo, estes resultados são coerentes com os de estudos brasileiros previamente publicados ${ }^{(14,15)}$, com a vantagem de ter aqui um número de indivíduos estudados quase dez vezes maior e uma quantidade significativamente maior de parâmetros estudados. Por fim, embora após ajustes não tenha havido uma maior prevalência de indivíduos com risco cardiovascular moderado/alto no grupo de pré-hipertensos, cabe considerar as limitações do escore de Framingham na estratificação de risco de indivíduos jovens, dado que, ao depender da idade, frequentemente subestima o risco cardiovascular nessa população ${ }^{(27)}$.

\section{CONCLUSÃo}

A pré-hipertensão é uma condição clínica altamente prevalente em nossa população e se associa de forma independente a síndrome metabólica, esteatose hepática, resposta hiper-reativa da pressão arterial durante o exercício e maior grau de inflamação subclínica. A adoção formal da pré-hipertensão como categoria diagnóstica permitiria rastrear e sensibilizar um grupo significativo de indivíduos de maior risco cardiovascular.

\section{REFERÊNCIAS}

1. Lewington S, Clarke R, Qizilbash N, Peto R, Collins R; Prospective Studies Collaboration. Age-specific relevance of usual blood pressure to vascular mortality: a meta-analysis of individual data for one million adults in 61 prospective studies. Lancet. 2002;360(9349):1903-13. Erratum in: Lancet. 2003;361 (9362):1060

2. Sociedade Brasileira de Cardiologia. VI Diretrizes Brasileiras de Hipertensão Arterial. Arq Bras Cardiol. 2010;95(1 supl.1):1-51.

3. Malta DC, Moura L, Souza FM, Rocha FM, Fernandes RM. Doenças crônicas não-transmissíveis: mortalidade e fatores de risco no Brasil, 1990 a 2006. In: Brasil. Ministério da Saúde. Secretaria de Vigilância em Saúde. Departamento de Análise de Situação de Saúde. Saúde Brasil 2008: 20 anos de Sistema Único de Saúde (SUS) no Brasil [Internet]. Brasília: Ministério da Saúde; 2009 [citado 2013 Jul 22]. Disponível em: http://portal.saude.gov.br/portal/ arquivos/pdf/saude brasil 2008 web 2011.pdf

4. Chobanian AV, Bakris GL, Black HR, Cushman WC, Green LA, Izzo JL Jr, Jones DW, Materson BJ, Oparil S, Wright JT Jr, Roccella EJ; National Heart, Lung, and Bloodlnstitute Joint National Committee on Prevention, Detection, Evaluation, and Treatment of High Blood Pressure; National High Blood Pressure Education Program Coordinating Committee. The Seventh Report of the Joint National Committee on Prevention, Detection, Evaluation, and Treatment of High Blood Pressure: the JNC 7 report. JAMA. 2003;289(19):2560-72. Erratum in: JAMA. 2003;290(2):197.

5. Qureshi Al, Suri MF, Kirmani JF, Divani AA, Mohamed Y. Is prehypertension a risk factor for cardiovascular disease? Stroke. 2005;36(9):1859-63.

6. Vasan RS, Larson MG, Leip EP, Evans JC, O’Donnell CJ, Kannel WB, et al. Impact of high-normalblood pressure on the risk of cardiovascular disease. $\mathrm{N}$ Eng J Med. 2001;345(18):1291-7.
7. Ortega KC, Ginani GF, Silva, GV, Mion D Jr. Pré-hipertensão: conceito, epidemiologia e o que falam as diretrizes. Rev Bras Hipertens. 2009;16(2):83-6.

8. Julius S, Nesbitt SD, Egan BM, Weber MA, Michelson EL, Kaciroti N, Black HR, Grimm RH Jr, Messerli FH, Oparil S, Schork MA; Trial of Preventing Hypertension (TROPHY) Study Investigators. Feasibility of treating prehypertension with an angiotensin-receptor blocker. N Engl J Med. 2006; 354(16):1685-97.

9. Pickering TG, Hall JE, Appel LJ, Falkner BE, Graves J, Hill MN, Jones DW Kurtz T, Sheps SG, Roccella EJ; Subcommittee of Professional and Public Education of the American Heart Association Council on High Blood Pressure Research. Recommendations for blood pressure measurement in humans and experimental animals: Part 1: blood pressure measurement in humans: a statement for professionals from the Subcommittee of Professional and Public Education of the American Heart Association Council on High Blood Pressure Research. Hypertension. 2005;45(1):142-61.

10. Craig $C L$, Marshall AL, Sjöström M, Bauman AE, Booth ML, Ainsworth BE, et al. International physical activity questionnaire: 12-country reliability and validity. Med Sci Sports Exerc. 2003;35(8):1381-95

11. Saunders JB, Aasland OG, Babor TF, de la Fuente JR, Grant M. Development of the Alcohol Use Disorders Identification Test (AUDIT): WHO Collaborative Project on Early Detection of Persons with Harmful Alcohol Consumption--II. Addiction. 1993;88(6):791-804.

12. Bellentani S, Saccoccio G, Masutti F, Crocè LS, Brandi G, Sasso F, et al. Prevalence of and risk factors for hepatic steatosis in Northern Italy. Ann Intern Med. 2000;132(2):112-7.

13. Alberti KG, Zimmet P, Shaw J. Metabolic syndrome--a new world-wide definition. A Consensus Statement from the International Diabetes Federation. Diabet Med. 2006;23(5):469-80.

14. IV Diretriz Brasileira sobre Dislipidemias e Prevenção da Aterosclerose. Departamento de Aterosclerose da Sociedade Brasileira de Cardiologia. Arq Bras Cardiol. 2007:88(supl.1):1-19.

15. Cassani R, Nobre F, Pazin AF, Schmidt A. Prevalência de fatores de risco cardiovascular em trabalhadores de uma indústria brasileira. Arq Bras Cardiol. 2009;92(1):15-21.

16. Silva DA, Petroski EL, Peres MA. Pré-hipertensão e hipertensão em adultos de Florianópolis: estudo de base populacional. Rev Saúde Publica. 2012; 46(6):988-98.

17. Singh JP, Larson MG, Manolio TA, O'Donnell CJ, Lauer M, Evans JC, et al. Blood pressure response during treadmill testing as a risk factor for new-onset hypertension. The Framingham heart study. Circulation. 1999;99(14):1831-6.

18. Nakashima M, Miura K, Kido T, Saeki K, Tamura N, Matsui S, et al. Exercise blood pressure in young adults as a predictor of future blood pressure: a 12-year follow-up of medical school graduates. J Hum Hypertens. 2004; 18(11):815-21.

19. Thanassoulis G, Lyass A, Benjamin EJ, Larson MG, Vita JA, Levy D, et al. Relations of exercise blood pressure response to cardiovascular risk factors and vascular function in the Framingham Heart Study. Circulation. 2012;125(23):2836-43.

20. Kshirsagar AV, Carpenter M, Bang H, Wyatt SB, Colindres RE. Blood pressure usually considered normal is associated with an elevated risk of cardiovascular disease. Am J Med. 2006;119(2):133-41.

21. Emerging Risk Factors Collaboration, Kaptoge S, Di Angelantonio E, Pennells L, Wood AM, White IR, Gao P, Walker M, Thompson A, Sarwar N, Caslake M, Butterworth AS, Amouyel P, Assmann G, Bakker SJ, Barr EL, Barrett-Connor E, Benjamin EJ, Björkelund C, Brenner H, Brunner E, Clarke R, Cooper JA, Cremer P, Cushman M, Dagenais GR, D’Agostino RB Sr, Dankner R, DaveySmith G, Deeg D, Dekker JM, Engström G, Folsom AR, Fowkes FG, Gallacher J, Gaziano JM, Giampaoli S, Gillum RF, Hofman A, Howard BV, Ingelsson E, Iso $H$, Jørgensen T, Kiechl S, Kitamura A, Kiyohara Y, Koenig W, Kromhout D, Kuller LH, Lawlor DA, Meade TW, Nissinen A, Nordestgaard BG, Onat A, Panagiotakos DB, Psaty BM, Rodriguez B, Rosengren A, Salomaa V, Kauhanen J, Salonen JT, Shaffer JA, Shea S, Ford I, Stehouwer CD, Strandberg TE, Tipping RW, Tosetto A, Wassertheil-Smoller S, Wennberg P, Westendorp RG, Whincup PH, Wilhelmsen L, Woodward M, Lowe GD, Wareham NJ, Khaw 
KT, Sattar N, Packard CJ, Gudnason V, Ridker PM, Pepys MB, Thompson SG, Danesh J. C-reactive protein, fibrinogen, and cardiovascular disease prediction. N Engl J Med. 2012;367(14):1310-20.

22. Blake GJ, Rifai N, Buring JE, Ridker PM. Blood pressure, C-reactive protein, and risk of future cardiovascular events. Circulation. 2003;108(24):2993-9. Erratum in: Circulation. 2007;115(20):e537.

23. Ndumele CE, Nasir K, Conceiçao RD, Carvalho JA, Blumenthal RS, Santos RD. Hepatic steatosis, obesity, and the metabolic syndrome are independently and additively associated with increased systemic inflammation. Arterioscler Thromb Vasc Biol. 2011;31(8):1927-32.
24. Duncan BB, Schmidt MI. The epidemiology of low-grade chronic systemic inflammation and type 2 diabetes. Diabetes Technol Ther. 2006;8(1):7-17.

25. Toprak A, Wang H, Chen W, Paul T, Ruan L, Srinivasan S, et al. Prehypertension and black-white contrasts in cardiovascular risk in young adults: Bogalusa Heart Study. J Hypertens. 2009;27(2):243-50.

26. McInnes G. Pre-hypertension: how low to go and do drugs have a role? $\mathrm{Br} \mathrm{J}$ Clin Pharmacol. 2012;73(2):187-93.

27. Akosah KO, Schaper A, Cogbill C, Schoenfeld P. Preventing myocardial infarction in the young adult in the first place: how do the National Cholesterol Education Panel III guidelines perform? J Am Coll Cardiol. 2003;41 (9):1475-9. 